\title{
The Evidence of Quasi-Free Positronium State in GiPS-AMOC Spectra of Glycerol
}

\author{
D. ZvezhinskiY ${ }^{a, b, *}$, M. Butterling ${ }^{c}$, A. WAgner $^{c}$, R. Krause-RehberG ${ }^{d}$ \\ AND S.V. STEPANOV ${ }^{a, b}$ \\ ${ }^{a}$ Institute for Theoretical and Experimental Physics, B. Cheremushkinskaya 25, 117218, Moscow, Russia \\ ${ }^{b}$ Moscow Engineering and Physics Institute, Dept. of Extreme States of Condensed Media, 115409, Moscow, Russia \\ ${ }^{c}$ Institute of Radiation Physics, Helmholtz-Zentrum, Dresden-Rossendorf \\ P.O. Box 510119, 01314, Dresden, Germany \\ ${ }^{d}$ Martin-Luther-University Halle-Wittenberg, Dept. of Physics, 06099, Halle, Germany
}

\begin{abstract}
We present the results of processing of age-momentum correlation spectra that were measured for glycerol by the gamma-induced positron spectroscopy facility. Our research has shown that the shape of experimental $s(t)$ curve cannot be explained without introduction of the intermediate state of positronium (Ps), called quasi-free Ps. This state yields the wide Doppler line near zero lifetimes. We discuss the possible properties of this intermediate Ps state from the viewpoint of developed model. The amount of annihilation events produced by quasi-free Ps is estimated to be less than $5 \%$ of total annihilations. In the proposed model, quasi-free Ps serves as a precursor for trapped Ps of para- and ortho-states.
\end{abstract}

DOI: 10.12693/APhysPolA.125.821

PACS: 78.70.Bj, 82.30.Gg, 34.80.-i, 34.80.Lx

\section{Introduction}

Among the set of positron annihilation methods, the age-momentum correlation (AMOC) technique yields the most complementary information about the fate of positron inside the investigated medium, that is its lifetime (or age) and the energy of annihilation gamma-quanta. Although these properties may tell researcher more information together than their separate measurements (i.e. a lifetime and Doppler spectra, respectively), the task of interpretation of 2-dimensional spectrum is practically more difficult. Recently we applied an approach based on such 2-dimensional interpretation of AMOC spectrum, that helped us to verify the diffusion-recombination model of Ps for the water, whose spectrum has been measured with gamma-induced modification of AMOC technique where slowing-down radiation gives birth to positron [1].

The lack of 2-dimensional approach is a relatively low count rate for each bin of the spectrum, that results in a large value for a relative error estimation. Additionally, the significant amount of bins in AMOC spectrum has no events, that makes it not reasonable to apply the chi-square approach for a fitting procedure.

To verify the Ps formation mechanism by means of interpretation of the gamma-induced positron spectroscopy-AMOC (GiPS-AMOC) spectra, we propose an alternative approach, which eliminates some of these issues. It has been applied to a glycerol at the set of

\footnotetext{
*corresponding author; e-mail: zmitja@yandex.ru
}

temperatures between 46 and $122^{\circ} \mathrm{C}$. Glycerol has been chosen as a convenient modeling substance because of its physical properties, that significantly change with temperature, namely viscosity, which is of greater interest for the current experiment, as it should influence the estimated time of medium reorganization in the presence of Ps and the growth of its bubble state.

\section{Experimental details}

The general description and advantages of GiPS-AMOC technique and its comparison with conventional technique were discussed previously [2]. We consider it mainly because of a perfect signal-to-background ratio and the absence of dissolved or sandwiched positron source. Before the experiment, the amount of glycerol (99.95\% grade) was heated in a vacuum oven in order to remove water before measurement. During the measurement the nitrogen has been bubbled through the glycerol, and the entry of new air from outside has been prevented. The supply system is located inside the irradiation chamber and exposed to bremsstrahlung, which gives birth to positrons inside the investigated volume. It is consisted of in-chamber kapton vessel, which was connected with plastic tubes to pump and heating systems, located outside the chamber. This scheme eliminated the appearance of positrons inside the most of auxiliary systems.

The processing of experimental spectra has been done as follows. From the AMOC spectrum, we extracted three experimental curves:

1. The lifetime spectrum $N(t)$, which is calculated as a projection of AMOC spectrum on time axis. For each $t$, the number of events was summed for events with energies of $E=m c^{2} \pm 5 \mathrm{keV}$. 
2. The Doppler spectrum $D(E)$, which is a projection of AMOC spectrum on energy axis. To obtain this dependence for each energy, the number of events was summed over all ages in the given spectrum.

3. The "sharpness" curve, or $s(t)$ curve, which is calculated at a given $t$ as a ratio of two sums of events, namely the number of events in a "narrow" range $(E=$ $m c^{2} \pm 0.7 \mathrm{keV}$ ) and the number of events in a "full" range (chosen as $E=m c^{2} \pm 3 \mathrm{keV}$ ). The significant part of the data is chosen between $-0.3 \mathrm{~ns}$ and $+6 \mathrm{~ns}$ with respect to "zero age" time.

As a measure of goodness-of-fit, the collective chi-square functional was chosen, which is introduced as a sum of squared residuals between models (see below) and corresponding experimental data, weighted with inverse error estimates. Its minimization yielded the results reported below.

\section{Gauss-exponential model with quasi-free Ps}

The main purpose of newly developed theoretical model is to describe the peculiarity of the data, particularly of $s(t)$ curve, where a peak in the region of "zero age" channel is observed for all temperatures of glycerol.

This feature means that at short lifetimes the shape of the Doppler spectrum varies from wide to narrow and then back to wide. We propose the following explanation to this phenomenon. The hot positron entering the investigated medium quickly loses its energy (this time is estimated as some picoseconds for a broad range of liquids), and then it becomes solvated by the surrounding medium at a time of about $10 \mathrm{ps}$ (estimates for electron trapping, see [3]). However, some part of these primary positrons form bounded state, which is called positronium (Ps). Because of electroneutrality, Ps interacts weaker with medium staying untrapped for some time. Such a delocalized electron-positron pair may be observed via its annihilation until its trapping occurs in the medium. To become trapped, it should find a structural defect with suitable size, and our estimates give the minimal radius of $2 \AA$ for this preexisting cavity. This search may take some time, which is expected to extract from the experiment. Thus, in the framework of the proposed model, the appearance of a wide component in the Doppler spectrum at short lifetimes is explained as annihilation of delocalized (or quasi-free) Ps.

Our current model is mainly similar to the one used in [4], especially in the part of physical origins of "juvenile" broadening and general way of model construction, except some details which will be highlighted below.

Let us show how quasi-free $\mathrm{Ps}_{\mathrm{s}}$ is introduced to the theoretical model. After the process of ionizational slowing down, positron either remains in a free state, or it becomes binded with any of intratrack electrons and forms quasi-free positronium state (qf-Ps). Then quasi-free state is trapped with the rate of $\kappa$ to localized $p$-Ps and $o-P s$ states, see Fig. 1 . In the proposed model we assume that this quasi-free Ps state possesses a Doppler

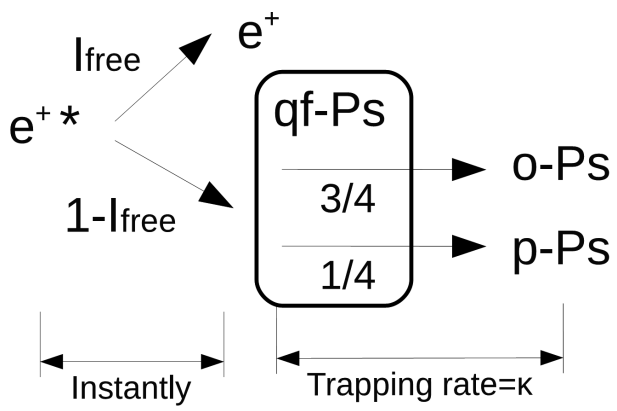

Fig. 1. The model of positron formation with quasi-free state.

line shape similar to that of free $\mathrm{e}^{+}$state, and its lifetime corresponds to $1 / \lambda_{\mathrm{e}+}$. This assumption was introduced to get rid of additional model parameters which make fitting less stable. We tried to fit these properties independently, but no notable difference was found between free values and equated to the values for $\mathrm{e}^{+}$.

The formulated model yields the following set of kinetic equations for $\mathrm{e}^{+}$and Ps concentrations:

$$
\begin{aligned}
& \frac{\mathrm{d} c_{\mathrm{qf}}(t)}{\mathrm{d} t}=-\kappa c_{\mathrm{qf}}-\lambda_{\mathrm{e}+} c_{\mathrm{qf}}, \quad c_{\mathrm{qf}}(0)=1-I_{\text {free }}, \\
& \frac{\mathrm{d} c_{\mathrm{e}+}(t)}{\mathrm{d} t}=-\lambda_{\mathrm{e}+} c_{\mathrm{e}+}, \quad c_{\mathrm{e}+}(0)=I_{\text {free }}, \\
& \frac{\mathrm{d} c_{\mathrm{p}-\mathrm{Ps}}(t)}{\mathrm{d} t}=0.25 \kappa c_{\mathrm{qf}}-\lambda_{\mathrm{p}-\mathrm{Ps}} c_{\mathrm{p}-\mathrm{Ps}}, \\
& \frac{c_{\mathrm{p}-\mathrm{Ps}}(0)=0}{\mathrm{~d} c_{\mathrm{o}-\mathrm{Ps}}(t)} \\
& c_{\mathrm{o}-\mathrm{Ps}}(0)=0.75 \kappa c_{\mathrm{qf}}-\lambda_{\mathrm{o}-\mathrm{Ps}} c_{\mathrm{o}-\mathrm{Ps}}
\end{aligned}
$$

Here, $I_{\text {free }}$ is the fraction of positrons that does not form Ps after the ionizational stopping, $c_{i}$ is the occupation number of $i$-th species, namely positron $\left(\mathrm{e}^{+}\right)$, qf-Ps, ortho- and para-Ps. This set of equations can be easily solved giving $c_{i}$. To obtain a lifetime spectrum from here, we sum items from the solutions of Eqs. (1)-(4) multiplied by corresponding annihilation rates

$$
\begin{aligned}
& N_{\text {theor }}(t) \simeq c_{\mathrm{qf}} \lambda_{\mathrm{e}+}+c_{\mathrm{e}+} \lambda_{\mathrm{e}+}+c_{\mathrm{p}-\mathrm{Ps}} \lambda_{\mathrm{p}-\mathrm{Ps}} \\
& \quad+c_{\mathrm{o}-\mathrm{Ps}} \lambda_{\mathrm{o}-\mathrm{Ps}} .
\end{aligned}
$$

We used the regions of $m c^{2} \pm 0.7 \mathrm{keV}$ and $m c^{2} \pm 3 \mathrm{keV}$ to calculate $s(t)$ curve from given binned AMOC experimental data $C\left(E_{i}, t_{j}\right)$ by summing it in these regions for given $t_{j}$, correcting sums on background contribution, and then dividing corrected sum for narrow range by that for wide range.

The fraction of background events is obtained by means of the following procedure. At first, the Doppler spectrum is fitted with Gaussian components and a stepwise curve as an additive background. After fitting the total proportion of background events is obtained as well as parameters defining the shape of background component.

The equation for theoretical model for $s(t)$ is derived from Eq. (5) by taking into account that every its item 
should be multiplied by the integral of the Doppler line with Gaussian shape, taken in the range of $m c^{2} \pm 0.7 \mathrm{keV}$ for numerator and $m c^{2} \pm 3 \mathrm{keV}$ for denominator

$$
\begin{aligned}
& s(t)=\left[\left(c_{\mathrm{qf}}+c_{\mathrm{e}+}\right) \lambda_{\mathrm{e}+} H_{1}\left(\sigma_{\mathrm{e}+}\right)+c_{\mathrm{p}-\mathrm{Ps}} \lambda_{\mathrm{p}-\mathrm{Ps}}\right. \\
& \left.\quad \times H_{1}\left(\sigma_{\mathrm{p}-\mathrm{Ps}}\right)+c_{\mathrm{o}-\mathrm{Ps}} \lambda_{\mathrm{o}-\mathrm{Ps}} H_{1}\left(\sigma_{\mathrm{o}-\mathrm{Ps}}\right)\right] \\
& \quad /\left[\left(c_{\mathrm{qf}}+c_{\mathrm{e}+}\right) \lambda_{\mathrm{e}+} H_{2}\left(\sigma_{\mathrm{e}+}\right)\right. \\
& \left.\quad+c_{\mathrm{p}-\mathrm{Ps}} \lambda_{\mathrm{p}-\mathrm{Ps}} H_{2}\left(\sigma_{\mathrm{p}-\mathrm{Ps}}\right)+c_{\mathrm{o}-\mathrm{Ps}} \lambda_{\mathrm{o}-\mathrm{Ps}} H_{2}\left(\sigma_{\mathrm{o}-\mathrm{Ps}}\right)\right] .
\end{aligned}
$$

Here $H_{i}(\sigma)=\int_{m c^{2}-\Delta_{i}}^{m c^{2}+\Delta_{i}} \exp \left(-\frac{\left(E-m c^{2}\right)^{2}}{2 \sigma^{2}}\right) /(\sqrt{2 \pi} \sigma) \mathrm{d} E$, where $\Delta_{1}=0.7 \mathrm{keV}$ and $\Delta_{2}=3 \mathrm{keV}$.

To construct the model for the Doppler spectrum, the annihilation contribution of corresponding Gaussian components should be computed for "free" positrons, ortho- and para-positronium. These fractions, denoted as $h_{i}$, are easily derived from the solutions of (1)-(4). Now we obtain the expression for the Doppler spectrum

$$
D_{\text {theor }}(E)=\sum_{i=1}^{3} \frac{h_{i}}{\sqrt{2 \pi} \sigma_{i}} \exp \left(\frac{\left(E-m c^{2}\right)^{2}}{2 \sigma_{i}^{2}}\right)+B(E) \text {. }
$$

The background component $B(E)$ has been previously fitted to processed spectrum with a stepwise function.

The resolution function for energy detector is taken into account by presuming its Gaussian shape, and, given that the shape of the Doppler components is the same, the standard deviation which enters Eq. (7) should be calculated as: $\sigma_{i}=\sqrt{\sigma_{\mathrm{rf}}^{2}+\sigma_{i, \mathrm{unc}}^{2}}$. The parameter of resolution function $\sigma_{\mathrm{rf}}$ is found from independent experiments and $\sigma_{i \text {,unc }}$ is uncorrected standard deviation for $\mathrm{e}^{+}, p-\mathrm{Ps}$, and $o$-Ps Doppler components.

\section{Results}

The collective fit of three theoretical models (5), (7), (6) to corresponding experimental data resulted in a set of obtained model parameters, that are discussed in this section. As a reference data for comparison with new results, we used a 3-exponential decomposition of lifetime spectra measured by Duplâtre and reported in [5].

Annihilation rates for para- and ortho-positronium were parameterised via a contact density parameter $\eta(T)$ (e.g. see [6]), that was not fixed because we were unable to find its value for glycerol in the available literature. The obtained values of $\eta$ are about $0.65 \pm 0.03$ without having any noticeable temperature trend. The lifetime of free $\mathrm{e}^{+}$and $\mathrm{qf}-\mathrm{Ps}$ is found to be $0.36 \pm 0.01 \mathrm{~ns}$ through all measured temperatures. The dependence for lifetimes of ortho-positronium on temperature is drawn in Fig. 2. It is in a good agreement with the reference data. The differences between them may be caused by fixing the formation ratio of $o$-Ps and $p$-Ps in Eq. (3), (4) to 3/1 in the present work, whereas it was obtained as a free parameter from the lifetime data. The lifetime of $o$-Ps shows the falling trend which is similar to the previously reported results. This behaviour is discussed in [5] and
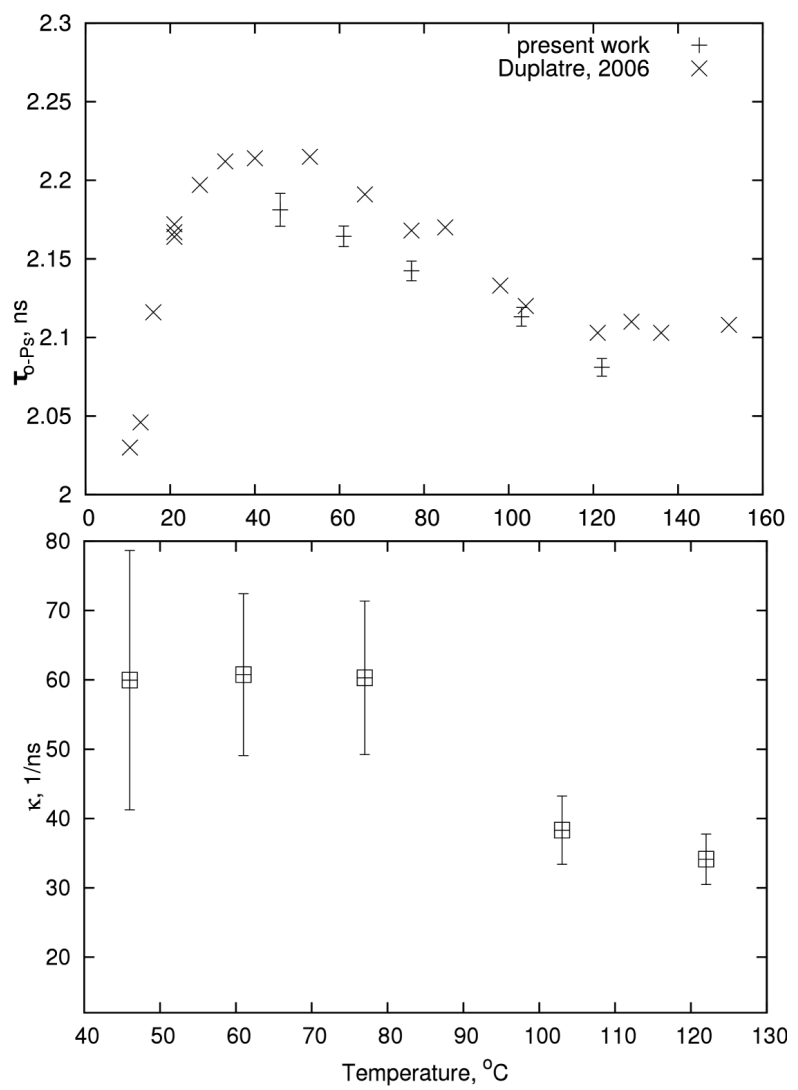

Fig. 2. Temperature dependence of $\tau_{\mathrm{o}-\mathrm{Ps}}$ (top) and trapping rate $\kappa$ (bottom) for glycerol.

it is caused by the neglecting of Ps chemical reactions in the current model.

Standard deviations $\sigma_{i}$ for the shape of the Doppler line are equal to $1.16 \pm 0.01 \mathrm{keV}$ for free $\mathrm{e}^{+}$and $1.02 \pm$ $0.02 \mathrm{keV}$ for $o$-Ps. They do not show any temperature trend as well. The values for $\sigma_{\mathrm{p}-\mathrm{Ps}}$ are varying from $0.3 \mathrm{keV}\left(46^{\circ} \mathrm{C}\right)$ up to $0.2 \mathrm{keV}\left(122^{\circ} \mathrm{C}\right)$.

The trapping rate $\kappa$ for quasi-free Ps turns out to be in the range of $35-601 / \mathrm{ns}$ (Fig. 2), which corresponds to the time of $16-30 \mathrm{ps}$. These values are close to the values obtained in [4], where they have the meaning of Ps slowing down times in condensed state media. It should be interesting to compare these estimates to the time of Ps bubble growth. According to estimations proposed by Mikhin et al. [7], it varies from $6 \mathrm{~ns}$ at $50^{\circ} \mathrm{C}$ to $1 \mathrm{~ns}$ at $70^{\circ} \mathrm{C}$, which is more than obtained values for $1 / \kappa$. This means that the limiting stage for Ps trapping is the search of an "empty" space in the medium, and not the Ps bubble growth, which should be large enough to allow the trapping of Ps.

Annihilation fractions $h_{i}$ drawn in Fig. 3 are compared to corresponding intensities from exponential decomposition. We observe a good agreement with reference data for $o$-Ps. The integral annihilation fraction for $p$-Ps slightly differs from the estimates obtained from lifetime spectra. The estimates of errors for parameters, drawn 


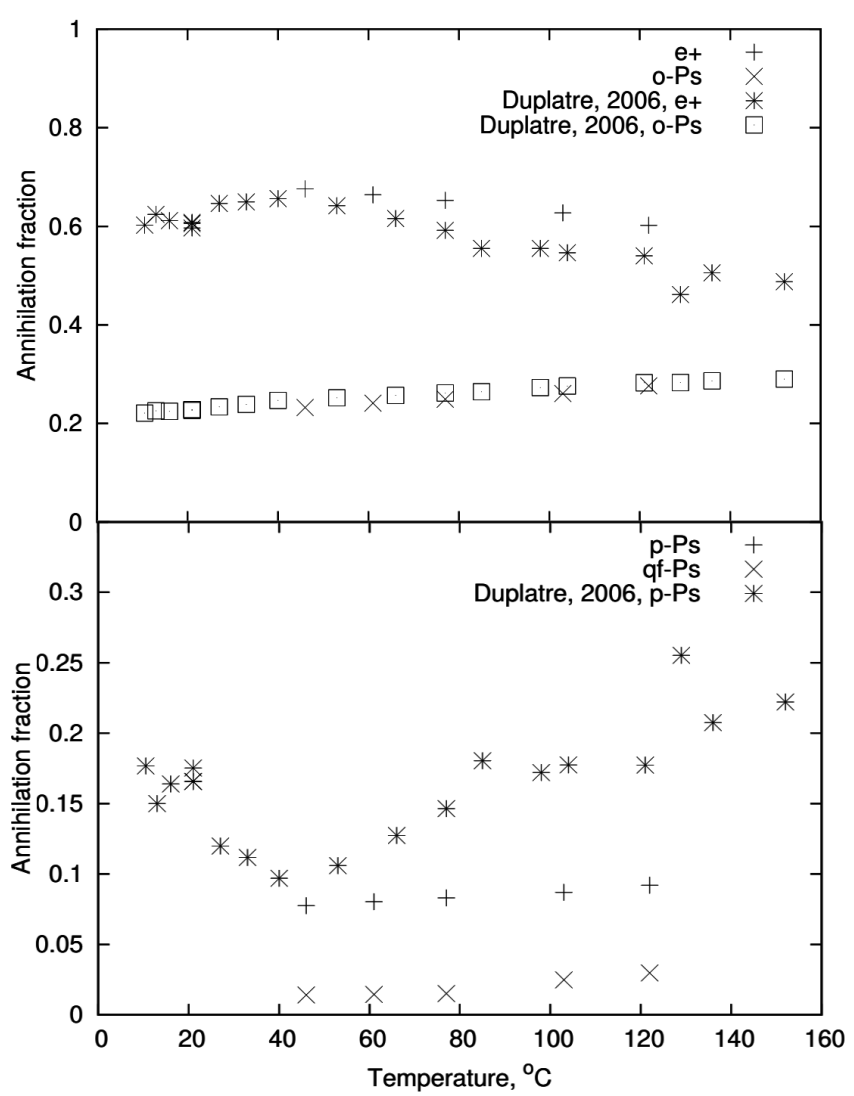

Fig. 3. Temperature dependence of annihilation fractions for glycerol.

in Fig. 3, are calculated with standard error propagation formula and their value does not exceed the size of marker. Errors for adjustable parameters are calculated by minuit routine [8], which is used for optimization of collective chi-square function.

\section{Conclusions}

With the currently developed theoretical model, we have described three experimental dependences, which are Doppler, lifetime spectra and $s(t)$ parameter. We have shown that it is necessary to account the presence of a delocalized Ps state at early stages of Ps interaction with the medium. In other words, the trapping rate $\kappa$ for that delocalized state, named quasi-free Ps, has finite value and the contribution to annihilation produced by qf-Ps differs from zero outside the range of estimated errors. Though the shape of the hump feature in $s(t)$ curve can be potentially changed by instrumental effects and data processing (e.g. see [9]), we should note that the presented way of AMOC spectrum interpretation should not substitute the complementary analysis of this data, including the extended analysis of background components as well as radiolysis mechanisms taken into account.

\section{Acknowledgments}

The work of D.S. Zvezhinskiy and S.V. Stepanov is supported by Russian Fund for Basic Research (grant no. 11-03-01066).

\section{References}

[1] D.S. Zvezhinskiy, M. Butterling, A. Wagner, R. Krause-Rehberg, S.V. Stepanov, J. Phys., Conf. Ser. 443, 012057 (2013).

[2] M. Butterling, W. Anwand, T.E. Cowan, A. Hartmann, M. Jungmann, R. Krause-Rehberg, A. Krille, A. Wagner, Nucl. Instrum. Methods Phys. Res. B 269, 2623 (2011).

[3] J. Bonin, I. Lampre, P. Pernot, M. Mostafavi, J. Phys. Chem. A 112, 1880 (2008).

[4] H. Schneider, A. Seeger, A. Siegle, H. Stoll, P. Castellaz, J. Major, Appl. Surf. Sci. 116, 145 (1997).

[5] S. Stepanov, D. Zvezhinskii, G. Duplatre, V. Byakov, V. Subrahmanyam, Mater. Sci. Forum 607, 260 (2009).

[6] S.V. Stepanov, D.S. Zvezhinski, G. Duplâtre, V.M. Byakov, Yu.Yu. Batskikh, P.S. Stepanov, Mater. Sci. Forum 666, 109 (2011).

[7] K.V. Mikhin, S.V. Stepanov, V.M. Byakov, High Energy Chem. 39, 36 (2005).

[8] F. James, CERN Program Library Long Writeup D506: MINUIT Function Minimization and Error Analysis, Reference Manual, http://wwwasdoc.web. cern.ch/wwwasdoc/minuit/minmain.html .

[9] T. Hirade, Radiat. Phys. Chem. 76, 84 (2007). 